\title{
interações \\ LA CIRCULARIDAD HERMENÉUTICA ENTRE EL UNUM Argumentum de SAN ANSELMO Y EL PROBLEMA DE DIOS EN ZUBIRI
}

\author{
A CIRCULARIDADE HERMENÊUTICA ENTRE O UNUM \\ Argumentum de Santo Anselmo e o Problema \\ DE DEUS EM ZUBIRI
}

\author{
THE HERMENEUTIC CIRCULARITY BETWEEN THE UNUM \\ Argumentum of SAINT ANSELM AND THE Problem \\ OF GOD IN ZUBIRI
}

\section{Jose Alfonso Villa Sanchez}

\begin{abstract}
RESUMEN
La presunción de este estudio es que entre la tesis del Unum argumentum de San Anselmo y la tesis de Zubiri sobre el problema de Dios- el hombre está vertido desde su estructura radical al @eós -, hay una relación hermenéutica circular. Con los recursos que ofrece este tipo de argumentación, aplicada a la relación que puede haber entre ambos autores, es posible poner al descubierto una continuidad intelectiva entre el argumento de San Anselmo y el concepto de realidad de Zubiri, conservando la estructura formal de la tesis del primero. Ambas tesis pueden complementarse, en una relación bidireccional, como apoyo para una reflexión filosófica más comprensora sobre el problema de Dios. La posibilidad de esa complementación pasa por la afirmación en Zubiri de que Dios es la realidad fundamental; y no el ser supremo, ni mucho menos un ente.

Palabras Clave: Dios. San Anselmo. Unum argumentum. Zubiri. Realidad fundamental. Religación.

\section{RESUMO}

O pressuposto deste estudo é que entre a tese do Unum argumentum de San Anselmo e a tese de Zubiri sobre o problema de Deus-o homem é esvaziado de sua estrutura radical para os @eós -, isto é, há uma relação hermenêutica circular. Com os recursos oferecidos por esse tipo de argumentação, aplicados à relação que possa existir entre os dois autores, é possível revelar uma continuidade intelectual entre a argumentação de San Anselmo e a concepção de realidade de Zubiri, preservando a estrutura formal da tese de primeira. Ambas teses podem ser complementadas, em uma relação de mão dupla, como suporte para uma reflexão filosófica mais abrangente sobre o problema de Deus. A possibilidade dessa complementação passa pela afirmação em Zubiri de que Deus é a realidade fundamental; e não o ser supremo, muito menos uma entidade.
\end{abstract}

\footnotetext{
${ }^{*}$ Doctor en Filosofía por la Universidad Iberoamerican de la Ciudad de México. Profesor de la Universidad Michoacana de San Nicolás de Hidalgo, Michoacán, México. ORCID: 00oo-0002-3254-0613. E-mail: jose.villa@umich.mx.
} 
Palavras-chave: Deus. Santo Anselmo. Unum argumentum. Zubiri. Realidade fundamental. Religião.

\begin{abstract}
This study presumes that between the thesis of the Unum argumentum of San Anselmo and the thesis of Zubiri on the problem of God-man is emptied from his radical structure to the $\Theta \varepsilon$ co-, there is a circular hermeneutical relationship. With the resources offered by this type of argument, applied to the relationship that may exist between the two authors, it is possible to uncover an intellectual continuity between San Anselmo's argument and Zubiri's concept of reality, preserving the formal structure of the former's thesis. Both theses can be complemented, in a two-way relationship, as support for a more comprehensive philosophical reflection on the problem of God. The possibility of this complementation passes through the affirmation in Zubiri that God is the fundamental reality; and not the supreme being, much less an entity.

Keywords: God. Saint Anselm. Unum argumentum. Zubiri. Fundamental reality. Religion.
\end{abstract}

\title{
I INTRODUCCION
}

La hipótesis de este trabajo sostiene que la tesis del unum argumentum, de San Anselmo -Dios es “[...] algo mayor que lo cual nada puede pensarse [...]” (SAN ANSELMO DE CANTERBURY, 2009, p. 79)-, tiene desde la tesis de el problema de Dios - Dios es un problema para el hombre-, tal como es planteada por Zubiri (2012, p. 3-23), unas posibilidades de comprensión mayores que las tenidas hasta el momento en la tradición, en virtud de que Dios es entendido por Zubiri como la realidad fundamental; no como un ente sustancial, y ni siquiera como el ser absoluto.

Dejar indicada la circularidad hermenéutica, y de reciprocidad, que se ofrece entre el unum argumentum y el problema de Dios precisa varios rodeos reflexivos y conceptuales, el más importante de los cuales es la comprensión de la realidad como algo de suyo, no como cosa ni como piélago de cosas. Este rodeo, sin embargo, debe estar precedido por la correcta comprensión del argumento de San Anselmo en su propio contexto.

\section{ARGUMENTACIÓN HERMENÉUTICA}

¿Cuál es el contexto del unum argumentum en San Anselmo? ¿y por qué ese argumento, más que una demostración, es una argumentación hermenéutica? Dado que una argumentación hermenéutica se mueve de manera circular entre varios elementos, y que ninguno de ellos guarda preeminencia -ni ontológica, ni epistemológica, ni cronológicasobre los demás, ¿cuáles son esos otros elementos con los que el unum argumentum está en 
relación de circularidad, como condición de posibilidad para su correcta y apropiada comprensión? Es preciso que esos otros elementos sean destacados.

He aquí el párrafo con el que da inicio el proemio del Proslogion, la obra de San Anselmo de Canterbury (1033-1109), cuyo célebre argumento ensambla perfectamente, según la hipótesis de esta meditación, con las tesis sobre el problema de Dios, contenidas en El Hombre y Dios, obra señera de Xavier Zubiri:

Luego de haber publicado, cediendo a los ruegos de algunos hermanos, un opúsculo como ejemplo de meditación sobre la razón de la fe por parte de una persona que, razonando calladamente consigo misma, investiga lo que no conoce, considerando que ese opúsculo estaba compuesto por un encadenamiento de muchos argumentos, comencé a preguntarme si quizá se pudiese encontrar un único argumento [unum argumentum] que no necesitase para su prueba [probandum] de ningún otro más que de sí, y que bastara por sí solo para asegurar [astruendum] que Dios es de manera verdadera, que es el sumo bien que no necesita de ningún otro y del que necesitan todas las cosas para ser y para ser buenas; $y$ todo lo que creemos acerca de la sustancia divina. (SAN ANSELMO DE CANTERBURY, 2009, p. $71)$.

¿Cuál es ese argumento único, capaz de intelectivamente cimentar y construir (astruendum) lo que la fe confiesa? Antes de que sea formulado debe repararse en el modo como quedan enunciadas racionalmente las verdades confesadas por la fe, porque es desde la fórmula de esta enunciación no, por ejemplo, desde la fórmula nicenoconstantinopolitana - desde la que aquel argumento se ofrece como prueba y constructo intelectual: “[...] a) que Dios es de manera verdadera, b) que es el sumo bien (1) que no necesita de ningún otro (2) y del que necesitan todas las cosas para ser y para ser buenas; y c) todo lo que creemos acerca de la sustancia divina.” (GONZÁLEZ FAUS, 1976, p. 417-463). ${ }^{1}$ Ahora sí: de ese Dios de la fe puede y debe decirse, con toda propiedad, desde la inteligencia sentiente propuesta por Zubiri, que es “[...] algo mayor que lo cual nada puede pensarse [...]” (SAN ANSELMO DE CANTERBURY, 2009, p. 78), tal como queda consignado el unum argumentum en el capítulo II del Proslogion.

El unum argumentum no tiene como propósito, para San Anselmo, demostrar que Dios existe; aunque una venerable tradición- que pasa por Santo Tomás de Aquino, Kant y llega hasta el propio J. L. Marion²- así haya leído este proemio y el capítulo II del Proslogion. La verdadera intención de San Anselmo no es otra sino mostrar que el acto de

\footnotetext{
${ }^{1}$ Destaca que no haya aquí rastros directos del símbolo niceno-constantinopolitano, y que más bien sean componentes de cepa neoplatónica los que alimentan el espíritu de San Anselmo (GONZÁLEZ FAUS, 1976).

2 Dice J. L. Marion que "[...] desde sus primeras páginas, el Proslogion le asigna al argumento un único objetivo, a saber, demostrar que Dios es el 'bien supremo' que no necesita de ningún otro bien y que todos necesitan para ser y ser buenos (summum bonum nullo alio indigens, et quo omnia indigent ut sint et bene sint; Proemio, 93)." (MARION, 2007, p. 200).
} 
fe del creyente en el Dios cristiano no es un acto vacuo ni absurdo, ilógico e irracional; que el acto de fe, que es más un acto afectivo y de la voluntad, puede tener una cierta continuidad, sin sobresaltos, con una particular lógica de la inteligencia. Y que esa continuidad, una vez que es establecida, observa un movimiento de circularidad con la confesión afectiva de fe, un movimiento de mutua compenetración y apoyo.

Ahora bien, si el unum argumentum no tiene pretensiones de ser demostrativo- ni probandum, ni astruendum, equivalen, en sentido estricto, a demostrar-, tampoco puede ser catalogado como un argumento ontológico, es decir, un argumento que pretendiera demostrar que Dios es, que Dios existe: los que ya creen en Dios- entre aquellos destinatarios del texto de Anselmo- no precisan que les sea demostrada su existencia; y los que no creen en el Dios cristiano- tanto como los que no creen en Dios alguno- tampoco están buscando un argumento para modificar su creencia. Se trata en el Proslogion, más bien, de una argumentación hermenéutica, que se mueve circularmente, 3 entre la fe cristiana que se profesa- con fuerte sabor neoplatónico y la voluntad por la intelección correcta del contenido de esa fe, condensado en la clásica fórmula de que Dios es "[...] algo mayor que lo cual nada puede pensarse [...]" (SAN ANSELMO DE CANTERBURY, 2009, p. 78). El primer destinatario de esta argumentación hermenéutica no es, desde luego - aunque pudiera parecer lo contrario, el otro distinto de sí: sea el insensato, el no creyente, o incluso el creyente, sea que busque pruebas o no para su fe. Al primero que complace esta argumentación, porque es al que le deja paz interior, es al que necesitaba buscarla, y encontrarla. Por eso puede pensar que presta un buen servicio a quienes están en la misma búsqueda, pero dudosamente a los demás. El creyente Anselmo ha encontrado una fórmula racional que ensambla perfectamente con la enunciación, también racional, de su fe.

Ha de señalarse, sin embargo, que no hay- y no puede haber- una continuidad deductiva entre el acto de la fe que se profesa y la fórmula lógica de la intelección que condensa racionalmente esa confesión de fe. Que no haya, sin embargo, continuidad deductiva no excluye toda continuidad intelectiva entre ambas esferas, porque la deducción demostrativa es sólo una forma- la más sencilla, por certo - de las muchas que tiene la intelección en tanto razón, según lo muestra Zubiri (1983, p. 17-91). La circularidad que hay entre lo que se cree (cualquiera que sea la creencia: lo que es tenido por bueno, por justo, por divino, etc.) y la manera como se comprende lo creído, precisa un modo de intelección hermenéutico, mayor en su complejidad a las deducciones más o menos lineales. La fe de un

3 Cfr. para el problema del círculo hermenêutico verifique (HEIDEGGER, 2003, p. 166-189). 
cristiano como San Anselmo, que busca afianzarse, comprender e inteligir- no demostrar, ha encontrado en la fórmula “[...] algo mayor que lo cual nada puede pensarse [...]" (SAN ANSELMO DE CANTERBURY, 2009, p. 78). La más alta intelección en relación a los contenidos de esa fe: la tarea será interpretarla para comprender mejor tanto la fe como la intelección (HEIDEGGER, 2003, p. 172); pero sobre todo la relación circular en la que mutuamente quedan comprendidas como un acto humano que aporta su riqueza a la constitución densa del sí mismo como otro (RICOEUR, 1996, p. 11-40), dado que ni la fe ni la intelección son actos de un yo individual, aislado, constituido como tal con anterioridad a la realización de su acto.

\section{EL PRINCIPIO DE LA CIRCULARIDAD}

La circularidad entre realidad e intelección de la realidad-propugnada por Zubiri (1980, p. 9-15) - debe ser elevada a principio fundamental. San Anselmo se inscribe en esa tradición política y piadosa, que remonta al profeta Isaías (Is. 7, 9)- y que tiene en San Agustín al más grande de sus pilares -, según la cual para entender quién es Dios (YHWH), qué es Dios, hay que creer en él, tener fe en que es lo que es, afianzarse en ello: tener fe, creer, es también una forma de inteligir; y no solo en relación a Dios. Aunque el contexto histórico y de ideas del profeta Isaías no es el de los cristianos de los primeros siglos y, por tanto, el contenido de las creencias y de lo que ha de entenderse es diferente, pues media entre tanto, además, la interpretación de la Vulgata,4 sobre el texto de Isaías, el presupuesto de la relación circular que se da entre lo que se cree y la necesidad de la correcta intelección de esa fe es la misma: lo que un momento aporta al otro se lo ofrece desde el interior mismo del círculo al que ambos momentos pertenecen. La esfera única de la fe-intelección- sitz im Leben del cristianismo durante su primer milenio- va a iniciar su desdoblamiento en esferas separadas- a la postre distintas y, finalmente contrapuestas- el siglo siguiente al de San Anselmo, por obra de las tesis de Averroes (GILSON, 2005, p. 79-92). De modo que el principio metodológico según el cual la fe busca la mejor intelección de sus contenidos es tenido, al menos hasta San Anselmo, por principio incuestionable. Pasar por alto este principio, ignorarlo, tratarlo con displicencia, minimizarlo, amputarle al círculo alguno de sus momentos, comprenderlo de manera equivocada, etc., son algunas de las faltas que se han cometido- ya desde Guanilo- en la recepción del Proslogion. Es preciso, pues,

\footnotetext{
4 Isaías $(7,9)$, que literalmente diría: "Si no os afirmáis en mí, no seréis firmes [...]”; la Vulgata traduce del siguiente modo: “[...] Si no creéis, no comprenderéis.” (Cfr. ZUBIRI, 2015, p. 9-10).
} 
recuperar en algunos momentos fundamentales, al menos, el principio metodológico de la circularidad entre fe e intelección de la fe, que está a la base de toda la filosofía y la teología de San Anselmo.

¿Cuáles son algunos de esos ejes contextuales, en los que gira con naturalidad la esfera única de la fe-intelección en el mundo del siglo XI? Uno de ellos es la vida de oración y trabajo de un monje benedictino: ora et labora, ideal de la vida cristiana de la época. Así que no debe extrañar que una oración cristiana, de súplica humilde, imbuida de espíritu neoplatónico (Cfr. SAN ANSELMO DE CANTERBURY, 2009, p. 78), sea la que pone marco afectivo al esfuerzo racional exhaustivo de San Anselmo por dar con unum argumentum que asegure y fundamente (astruendum) "[...] todo lo que creemos acerca de la sustancia divina." (SAN ANSELMO DE CANTERBURY, 2009, p. 71). He aquí el talante de esa oración:

Que pueda levantar los ojos hacia tu luz, sea desde la lejanía o desde la profundidad. Enséñame a buscarte, y muéstrate a quien te busca, porque ni puedo buscarte, a menos que tú me enseñes; ni encontrarte, a menos que te muestres. Te buscaré deseándote, para desearte buscándote. Te encontraré amándote, para amarte encontrándote. Lo reconozco, Señor, y te doy las gracias, porque has creado en mí esta imagen tuya, para que, acordándome de ti, te piense y te ame. Pero está tan borrada por el roce de los vicios, está tan oscurecida por el humo de los pecados, que no puede hacer aquello para lo cual fue hecha, a menos que tú la renueves y la reformes. No aspiro, Señor, a penetrar tu profundidad, porque de ningún modo comparo con ella mi entendimiento; pero deseo entender de algún modo tu verdad, que cree y ama mi corazón. Porque tampoco busco entender para creer, sino que creo para entender. Pues también creo esto: que "si no creo, no entenderé." (SAN ANSELMO DE CANTERBURY, 2009, p. 78).

Las cosas verdaderas no solamente se las entiende sino que, sobre todo, se las ama, y se cree en ellas; por eso se las puede entender mejor. Ni la existencia de Dios, ni su inexistencia, tienen cabida, en sentido estricto, sólo al interior de los mejores argumentos demostrativos, estrictamente racionales y lineales. Y confesada -no demostrada- su existencia, lo mismo debe decirse de su naturaleza. Quienes han intentado, a lo largo de la historia de las ideas, tratar el problema de Dios por el camino de la demostración deductiva han hecho entrar sus tesis en callejones sin salida, devenidas no pocas veces en posiciones ideológicas y dogmáticas. Esto lo sabe cualquier filósofo -cristiano, teísta o ateo-, cualquier teólogo y, en general, cualquiera que haya reflexionado un poco sobre el asunto. De modo que el papel de la inteligencia en relación al problema del ser de Dios, de su existencia y su naturaleza, ha de buscarse por una vía diferente a la de la demostración5. ¿Cuál puede ser

\footnotetext{
5 Mariano Álvarez Gómez, en la presentación del Proslogion, editado y traducido por Julián Velarde, se equivoca desde el párrafo de entrada al atribuir al unum argumentum de San Anselmo intenciones demostrativas: “[...] Anselmo de Canterbury (1033-1109) es conocido e incluso célebre en el campo de la
} 
esa otra vía del saber, que eche a andar el problema de Dios por una ruta diferente a la demostración? La vía de la circularidad hermenéutica, fundada, no en la comprensión del Dasein, según la tesis de Heidegger (Cfr. 2003, p. 166-172), sino en la estructura de los actos de la inteligencia sentiente propuesta por Zubiri (1980, p. 166-172), cuyo acto radical consiste en el mero hacerse presente de la realidad en la intelección, fundamento de toda posibilidad de una razón que fuera demostrativa. En todo caso, la demostración va a demostrar sobre algo en lo que ya está instalada la inteligencia; la demostración no va a conocer, por primera vez, aquello que pretende demostrar.

Desandar el camino desde la razón, desde el cogitari, hasta la inteligencia sentiente, que le sirve de fundamento, lleva consigo desandar el camino de la entificación de Dios hacia su realidad: Dios no es un ente, ni siquiera el Ser. La reflexión que se ocupe de Dios y lo trate, aunque sea tácitamente, como un ente, ha errado el inicio mismo del camino; incluso aquella que lo hace esforzándose por tratarlo como el ser supremo no puede sino acarrear consigo los presupuestos con los que se entiende una sustancia y, vía ese camino, es inevitable que la realidad divina quede convertida en un súper ente, la cosa más grande que existe. Y ni la idea de cosa, ni la de cantidad superlativa, ni la de existencia, aplican para hablar con propiedad de la realidad de Dios, que no es algo, pero tampoco simplemente alguien. A la predicación de la grandeza cuantitativa respecto de ese ente que es Dios, y a la predicación de su existencia como un existente estar a la mano de lo que es superlativamente grande, sólo le quedarán los recursos de la analogía; una analogía que, por su propia naturaleza, se mueve en la precomprensión de la estructura jerárquica del ser como ente, que pretende ir, metodológicamente de manera injustificada, de lo más pequeño intramundanamente a lo más grande extramundanamente.

Pero Dios no es un ente, ni siquiera el ser supremo; ni está tampoco fuera de este mundo, ya sea como motor inmóvil (SANTO TOMÁS DE AQUINO, 2001, p. 107-113) o como epifanía del rostro (LÉVINAS, 2006, p. 201-261). ¿Qué puede decirse entonces sobre Dios? Que es, aquí y ahora, la realidad fundamental (ZUBIRI, 2012, p. 457-467); fundamento desde dentro, por tanto, del único mundo real que es este. ¿Y no hay en esta afirmación, de nueva cuenta, una recaída en esa manía que hace de Dios una cosa, un ente, un ser, una sustancia, un hombre divino? No; porque realidad no es cosa -ni ente, ni ser, ni sustancia. La realidad es la formalidad de ser de suyo, la formalidad de ser en propio tal o cual realidad. Dios es de suyo la realidad fundamental. La distinción de Zubiri entre realidad, ser y ente se

Filosofía por el argumento que formuló en el Proslogion (1077-1078) para demostrar tanto la existencia como la naturaleza de Dios." (GÓMEZ, 2009, p. 13). 
ofrece como la mejor alternativa en el camino para que el unum argumentum de San Anselmo se muestre en sus posibilidades más profundas, al mismo tiempo que en sus límites: Dios no es ni un ente frente a otros entes, ni tampoco el ser que queda subsumido en todo ser. San Anselmo ha tenido -después de una ardua y espinosa lucha interior- una genial intuición intelectiva (no meramente lógica, ni ontológica, ni existencial) para ponerle finalmente palabras a su fe, profunda y sincera: Dios es de suyo "[...] algo mayor que lo cual nada puede pensarse [...]" (SAN ANSELMO DE CANTERBURY, 2009, p. 78). Sin embargo, cuando quiere que esa prístina intuición pase por la estructura argumentativa y deductiva, para que su contenido sea mostrado con evidencia a aquellos hermanos de la Orden de San Benito que le han pedido ayuda con un ejemplo para aprender a meditar -lo cual es un ejercicio de la razón- sobre su fe, Anselmo se ve arrastrado hacia una serie de explicaciones ónticas que, entre sus propios hermanos, encuentra las primeras críticas y sospechas. Aunque todo benedictino suscriba la enseñanza de San Agustín de que hay que creer para entender (SAN AGUSTÍN, 1991, p. 73-74), no significa sin embargo que piense que la fe tenga que pasar necesariamente por las pruebas de la razón: algunos creen que es suficiente la fe sencilla que Dios le concede a cualquiera, y que el recurso a la razón no sólo no hace la fe más robusta sino que la debilita y hasta la pone en riesgo. Otros creen, de plano, que entre la fe cristiana y la razón griega (platónica, aristotélica, neoplatónica, etc.) no hay ni necesidad ni posibilidad de diálogo. Otros, como San Anselmo, piensan que, instalados en el territorio de la fe, no se deben ahorrar esfuerzos para comprender lo que ha sido recibido: que Dios es, de suyo, “[...] algo mayor que lo cual nada puede pensarse [...]” (SAN ANSELMO DE CANTERBURY, 2009, p. 78). No se trata de demostrar que Dios existe -icon lo que Anselmo tenga de precursor lejano de Descartes, Leibniz o Kant, está muy lejos de ese prurito demostrativo moderno!- sino de inteligir, de comprender, que Dios es "[...] como creemos, y [...] eso que creemos." (SAN ANSELMO DE CANTERBURY, 2009, p. 78). No se trata, pues, de inteligir si Dios es, ni siquiera de inteligir si es como creemos; se trata directamente de inteligir a) que es como creemos, y b) que es eso que creemos. Se trata de comprender al Dios en el cual se tiene ya puesta la confianza; y no de demostrar su existencia. Esto es lo sensato, lo que haría cualquier hombre piadoso, prudente y sabio; el insensato, aunque sea monje benedictino -precisamente por ello su insensatez es aún más atrevida-, piensa que la resolución de las dos condicionales, es decir, de si Dios es y de si es como lo dice la fe, son limitantes para entrar profundamente en el contenido de la fe. Pero es exactamente al revés: ir llanamente a la intelección y comprensión de la fe en Dios en tanto un Dios que es, guarda con la demostración de su existencia y de sus atributos una 
relación de circularidad comprensiva: habiendo creído se entiende mejor que Dios existe y el modo como existe. No al revés: el mejor argumento para demostrar que Dios existe no está jamás en el origen del don de la fe.

El hermano Guanilo, por cierto, es una vuelta de tuerca a la estulticia del insensato del salmo ${ }^{6}$ : este sólo dice en su corazón que Dios no es, que no existe: que quizá no haya Dios, que su ser no tiene la claridad que poseen las cosas a la luz del día; y la dubitación no abandona ese recinto de su interior. Pero Guanilo no trae esas cosas en su corazón sino en la cabeza, sembrándole vacilaciones en la mente; por eso puede hacer de ese titubeo, ya no una mera incertidumbre interior, sino una alocución, un alegato, y expresar contundentemente que de una idea cualquiera no se sigue con necesidad su existencia en el mundo extramental. Pero debe repararse en que este es ya otro problema respecto al de San Anselmo: pues no es lo mismo tratar de inteligir que Dios es " $[$...] como creemos, y [...] eso que creemos [...]" (SAN ANSELMO DE CANTERBURY, 2009, p. 78), que tratar de demostrar si es, si existe. Una es, pues, la preocupación de Anselmo -inteligir que Dios esy otra la que esboza Guanilo -inteligir si Dios es-. No es la primera vez en filosofía que se dice una cosa y se entiende otra. Mientras que Anselmo solo quiere inteligir lo que Dios es, Guanilo, y quienes siguieron por su misma estela, quieren demostrar si Dios es. El estado de ánimo que da origen a una y a otra intención es claramente distinto. Sin embargo, el estudio y la recepción del argumento de San Anselmo ha ido quedando aprisionado en la tradición por el corsé de la demostración, por el prurito de la autonomía de la razón. Pero no era ese el espíritu ni del Monologion ni del Proslogion.

Atrapados por la concepción neoplatónica de la jerarquía del ser, y por la comprensión de este como sustancia -cuyos extremos de riesgo en el caso de Dios son, por un lado, la antropomorfización y, por el otro, la disolución panteísta-, sin la distinción entre realidad, ser y ente, el diálogo entre San Anselmo y Guanilo, entre el creyente y el insensato, entre Anselmo y Kant, termina siendo un diálogo de sordos. Pues para que haya diálogo ha de poder mediarse entre las posiciones, han de poder mostrarse los presupuestos sobre los que los oídos se vuelven mutuamente indiferentes. El apoyo viene, de nueva cuenta, de la filosofía primera de Zubiri: el intelligere de San Anselmo, más que conocer algo que ignora, quiere leer (legere) por dentro (intus), desde el interior, aquello en lo que ya está, aquello que ya sabe de alguna manera, tal como Zubiri entiende que corresponde al papel de la inteligencia sentiente (ZUBIRI, 2015, p. 18). ¿En qué es en lo que ya está instalada la

\footnotetext{
6 “Dice en su corazón el insensato: ‘iNo hay Dios!'. Corrompidos están, de conducta abominable, no hay quien haga el bien.” (Sl 14, 1).
} 
inteligencia, de modo que no debe hacer por llegar a ello demostrándolo? La respuesta es que el hombre, por la inteligencia sentiente que le constituye esencialmente, está ya siempre instalado en la realidad. Eso en lo que ya se está es la realidad, momento radical y fundante de todo ser y, desde luego, del ente. El acto radical de la inteligencia consiste simple y modestamente en inteligir lo que hay, y tal como lo hay: lo real se hace presente en la inteligencia como siendo lo que ya es. El Dios sobre el que se quiere inteligir que es y como es no es, por principio, un desconocido; no es algo sobre lo que se va a pensar por primera vez. Al contrario: ya se lo conoce porque ya se cree en él, ya la vida y su sentido están en él afianzadas; y la creencia ( $\delta o ́ \xi \alpha$ ) es también un modo de conocimiento, un modo de hacerse presente en la inteligencia la realidad en tanto creída. ¿̇ué es, pues, más precisamente inteligir?

Inteligir consiste formalmente en aprender lo real como real [...]. Aquí real significa que los caracteres que lo aprehendido tiene en la aprehensión misma los tiene "en propio", "de suyo" [...]. No se trata de cosa real en la acepción de cosa allende la aprehensión, sino de lo aprehendido mismo en la aprehensión pero en cuanto está aprehendido como algo que es "en propio". Es lo que llamo formalidad de realidad. (ZUBIRI, 2015, p. 12).

$\mathrm{Ni}$ un inteligir que es en primer lugar demostración, ni un inteligir que debe alcanzar algo en lo que aún no está, son el recurso adecuado para entender el espíritu del argumento de San Anselmo. Porque si es verdad que los caracteres que la inteligencia aprehende de la realidad los aprehende en tanto que son algo de esa realidad -que es en propio lo que es, antes de ser aprehendida, pero en la aprehensión-, esto significa entonces que, en alguna medida, inteligencia y realidad se generan en el mismo movimiento. "Es que es imposible una prioridad intrínseca del saber sobre la realidad ni de la realidad sobre el saber [...]. No hay prioridad de lo uno sobre lo outro." (ZUBIRI, 1980, p. 10). Extrínsecamente parece como si la inteligencia se generara y se constituyera de manera paralela a la realidad; mirado el asunto con más detenimiento, sin embargo, se va aclarando que en el mismo movimiento en que ella va constituyéndose sucede lo propio con la realidad en tanto inteligida. El ojo se hace ojo viendo y el oído oyendo: y lo visto y lo oído, si bien son uno con el ojo y el oído, son de suyo realmente algo diferente al ojo y al oído7. Y dado que ver y oír son modos de inteligir, puede decirse que inteligencia y realidad "[...] son en su misma raíz estricta y rigurosamente congéneres.” (ZUBIRI, 1980, p. 10).

\footnotetext{
${ }^{7} \mathrm{Al}$ igual que Zubiri, también Merleau-Ponty se ha ocupado de este asunto (Cfr. MERLEAU-PONTY, 2000, p. 230).
} 


\section{EL ARGUMENTO: SU CONTEXTO Y SU INTELECCIÓN}

¿Cuál es el contexto más inmediato del unum argumentum? He aquí, completo, el breve capítulo II del Proslogion, titulado Que Dios es verdaderamente:

[a] Así, pues, Señor, tú que das entendimiento a la fe, concédeme a mí, en la medida en que tú sabes es útil, entender que tú eres, como creemos, y que eres eso que creemos. [b] Y, sin duda, creemos que tú eres algo mayor que lo cual nada puede pensarse. Pero ċy si no hay una tal naturaleza, puesto que "dijo el insensato en su corazón: no hay Dios" [Sal. 14,1 y 52, 1]. Pero, ciertamente, ese mismo insensato, cuando oye esto mismo que digo: "algo mayor que lo cual nada puede pensarse [cogitari]", entiende [intelligit] lo que oye; y lo que entiende está en su entendimiento, aún cuando no entienda que aquello es. En efecto, que una cosa sea en el entendimiento [intellectu] es algo diferente de entender que la cosa es. Pues cuando un pintor piensa de antemano [praecogitat] lo que va a hacer, lo tiene, ciertamente, en el entendimiento [intellectu], pero aún no entiende que sea lo que aún no ha hecho. Pero cuando él ya ha pintado, entonces, a la vez, lo tiene en el entendimiento y entiende que es lo que ya ha hecho. Por tanto, también el insensato está obligado a reconocer que, al menos en el entendimiento [intellectu], es algo mayor que lo cual nada puede pensarse [cogitari], porque cuando oye esto, lo entiende [intelligit]; y todo lo que se entiende es en el entendimiento. $\mathrm{Y}$, ciertamente, aquello mayor que lo cual nada puede pensarse no puede ser sólo en el entendimiento [intellectu]. Pues si es sólo en el entendimiento, puede pensarse que es también en la realidad, lo cual es mayor. Por tanto, si aquello mayor que lo cual nada puede pensarse [cogitari] es sólo en el entendimiento [intellectu], entonces eso mismo, aquello mayor que lo cual nada puede pensarse, es aquello mayor que lo cual nada puede pensarse. Pero ciertamente, esto no puede ser. Luego, existe, sin duda, algo mayor que lo cual nada puede pensarse, tanto en el entendimiento [intellectu] como en la realidad8. (SAN ANSELMO DE CANTERBURY, 2009, p. 78-79).

La primera oración de este tesoro del pensamiento le ofrece varias pistas a la reflexión. ¿Cuál es el antecedente a esa partícula consecuente del inicio? ¿qué presupone ese Así, pues, (Ergo), para la exposición y la argumentación que le sigue? Porque lo que viene a continuación -el unum argumentum - no se explica sólo y desde sí mismo, sino que recoge de esos antecedentes su pujanza. Los antecedentes inmediatos están en el capítulo I Incitación del espíritu a la contemplación de Dios-, y en el Proemio del propio Proslogion; y otros, un poco más distantes, aparecen en el Monologion (Cfr. SAN ANSELMO, 1980, p. 44-111).

Las primeras líneas del Proemio del Proslogion -que remiten a los motivos contextuales del Monologion- dicen que el autor pretendió ofrecer en este último un breve texto con un objetivo muy preciso: “[...] que sirviera de ejemplo de meditación de la razón de la fe.” (SAN ANSELMO DE CANTERBURY, 2009, p. 71). Las relaciones entre los

${ }^{8}$ Conviene tener ante la vista atenta la versión latina. 
contenidos de la fe cristiana, recibida gratuitamente, y la razón -de raigambre grecolatina, con la que aquella fe se trata de inteligir, de concebir, de entender, requiere meditación; no primeramente argumentación, ni demostración. ¿Cuál es la diferencia? ¿qué es lo propio de la meditación, en el marco de la rica tradición monacal cristiana, que remonta a las viejas escuelas estoicas, epicúreas y pitagóricas? En la meditación, la fe y su intelección se amoldan y conforman mutuamente; y se van acomodando así, sin violencia, sin que cada una rebase sus propios dominios, ni invada a la otra, en el interior de aquel espíritu que quiere progresar en este conocimiento, en esta experiencia. Pero -ioh paradoja!- el mutuo atemperamiento de fe y razón precisa silencio interior, y diálogo íntimo del alma consigo misma: sólo así las alocuciones que vengan estarán protegidas de caer en peroratas meramente exteriores, al servicio de vocingleros nocturnos, como gustará de decir Kierkegaard (Cfr. 2012, p. 42-43). Abandonarse a la meditación, en la que la inteligencia asiste, en una pasividad activa, al avenimiento entre fe y razón, a las medidas que una debe tomar respecto de la otra, requiere no sólo esfuerzo y decisión sino también pericia: meditar no es una tarea fácil, ni aunque se tengan momentos del día asignados para ello, como lo manda la regla de San Benito. ¿Cómo hacer para no convertir los valiosos lapsos asignados a la oración de meditación ni en agujeros del día, vacíos de progreso interior, ni tampoco en minutos de elucubraciones racionales, igualmente estériles para el crecimiento del espíritu? Mediante el silencio activo y el diálogo del alma consigo misma: internándose, decidida y calladamente, en el mundo de los misterios cristianos -más bien: dejándose invadir resueltamente por ese universo-, mientras el alma va intercambiándose de lugar entre el agente y el paciente respecto de la intelección que se le ofrece, respecto de la lectura que brota desde el interior de la inteligencia de la fe. En la meditación se le ofrece a la razón la fe en la que la inteligencia ya está; no a la que habrá de llegar.

¿Qué significa que la inteligencia ya está instalada en la fe; y que sólo por eso aparece la posibilidad de que esa fe sea ofrecida a la razón, para que mutuamente se conformen? Entre otras cosas, que inteligencia y razón no son lo mismo; que la razón es un momento fundado del despliegue de la inteligencia sentiente. El Ejemplo de meditación sobre el fundamento racional de la fe o Monologion, aquel pequeño libro que quería ser ejemplo de meditación, parece haber fracasado. ¿Por qué? Porque se olvidó precisamente de ser ejemplo de meditación e, inadvertidamente, se pasó del lado de esas prácticas de la razón que acumulan argumentos y, en ese acopio, sepultan el contenido de la fe y asfixian, o cuando menos ensucian gravemente, el diáfano hálito del alma. Los momentos para meditar deben ser momentos en los que el alma ha de disponerse de tal manera que pueda ser 
llevada, aquí, en vilo por la eternidad; mientras que los momentos para intentar arrancarle esquirlas de racionalidad a los misterios de la fe deben ser claramente distintos, aunque los empalmes y mutuos recubrimientos no sólo son inevitables sino siempre bienvenidos. Queriendo servir a los demás, directamente a los hermanos interesados -sólo a ellos-, pretendiendo ayudar para orientar durante los arduos momentos dedicados a la meditación, probablemente más bien se los distraiga; y lo que tendría que ser entrega al misterio, arrobamiento, se convierta en un mero y pesado ejercicio de atención lógica, cosa además que puede hacer -y quizá con más provecho para sí- incluso quien no tiene fe.

Después de aquel fracaso, es hora de recomponer el camino, de volver a la meditación. $\mathrm{Y}$, dado que se vuelve a la meditación meditando, “[...] comencé a preguntarme -dice San Anselmo- si quizá se pudiera encontrar un único argumento [...]” (SAN ALSELMO DE CANTERBURY, 2009, p. 71); un argumento que se bastara a sí mismo, que no precisara del lastre de otros argumentos. ¿Será posible encontrar, no en la naturaleza, ni en el mundo en el orden que tiene la naturaleza-, ni en el hombre en tanto cosa natural y mundana, sino en el interior del alma -interior que no es ni físico, ni cosmológico, ni siquiera antropológico- un testimonio intelectual que se baste a sí mismo: con la fuerza necesaria para fundamentar racionalmente "[...] todo lo que creemos acerca de la sustancia divina [...]" (SAN ALSELMO DE CANTERBURY, 2009, p. 71).

De los muchos detalles de la meditación de San Anselmo, en los que la reflexión crítica puede reparar, está la distinción -se puede presumir que ni inocente ni ingenua, aunque tampoco explícitamente reconocida- entre cogitare e intelligere, entre pensar y entender, según la versión castellana utilizada. Esa sutil distinción entre cogitar e inteligir, entre razón e inteligencia, es la que de por sí incluye en el círculo de la argumentación hermenéutica, expandiéndolo y enriqueciéndolo, la estructura de la inteligencia sentiente, tal como Zubiri la propone, con sus momentos de aprehensión primordial de realidad, logos sentiente y razón sentiente.

¿Dónde se ofrece el testimonio que autoriza la afirmación de que intelligere y cogitare se distinguen, al menos en el capítulo II del Proslogion, locus del unum argumentum? En el uso que San Anselmo hace de los dos verbos en el propio texto; y también más allá de este capítulo II. Cada vez que aparece la fórmula "[...] algo mayor que lo cual nada puede pensarse (cogitari) [...]” (SAN ALSELMO DE CANTERBURY, 2009, p. 78), el verbo es el mismo: cogitare. Y cuando se apela a que incluso el insensato entiende (intelligit) lo que se dice en esa fórmula, la forma verbal es intelligere. Si se apura un poco la distinción en el contexto, inteligir es un acto humano que el hombre no puede no hacer: simplemente porque 
lo entendido se hace presente en la intelección por la fuerza y el poder de su propia realidad. Mientras que, por el contrario, cogitare es un acto fundado en la intelección, que se lo puede llevar a cabo o no. Habiendo sido ya inteligida la realidad -habiéndose hecho presente ella, por su propia iniciativa, en la inteligencia sentiente-, se puede agitar (agitare) esa primaria intelección junto (co, cum, con) a otras intelecciones, y concebir entonces la fórmula “[...] algo mayor que lo cual nada puede pensarse [...]" (SAN ANSELMO DE CANTERBURY, 2009, p. 78). Ahora bien, la intelección que ha acompañado la búsqueda de esta fórmula, que la ha motivado, y que se siente satisfecha una vez que la ha encontrado, es que Dios es, y que es como creemos. De manera que, en la agitación intelectiva común que es el cogitare, no se buscaba lo que no se sabía; sino que se ha encontrado, en la agitación racional de unas cosas con otras, aquello de lo que ya se estaba en posesión por la esfera que, como unidad, conforman la fe y la intelección en el universo de un cristiano como San Anselmo.

El progreso de la filosofía no es obra de los individuos sino del pensamiento, que retoma otros pensamientos y les da continuidad, sumergiéndose con profundidad en la realidad. Zubiri es un filósofo cristiano del siglo XX y, como San Anselmo, también él se ocupa de pensar sobre el Dios de su fe. Aunque el contexto histórico y de ideas es otro, el reto para el creyente que se toma en serio pensar sobre los contenidos de la fe y, en última instancia, sobre Dios, es el mismo: que Dios no sea reducido a un ente más del mundo, así sea el ente ejemplar, fundamental. Y, en el caso específico de Zubiri, que tampoco sea reducido simplemente a ser, así sea el Ser Superior. "[...] Algo mayor que lo cual nada puede pensarse [...]" (SAN ANSELMO DE CANTERBURY, 2009, p. 78). Según la intuición de San Anselmo, se torna efectivamente una aporía si a toda costa se ha de identificar al Dios de la fe con un ente o un ser, por más que se purgue y purifique de contenidos impropios el concepto. No es en vano el esfuerzo por mantener la expresión en sentido neutro, cuidándose de llenarla con cualquier contenido porque, precisamente, al hacerlo dejaría de aportar para la comprensión correcta y verdadera de la realidad divina. Pero el unum argumentum tampoco es una mera categoría formal de la razón, porque Dios no es sólo un concepto vacío, ni tampoco una mera idea para la praxis, como piensa Kant (1997, p. 670). El unum argumentum es una categoría de realidad, un predicado sobre algo que es de suyo, y distinto, por tanto, al pensamiento que lo piensa; que es, en definitiva, real. Por eso la presunción es que la elipse racional, iniciada en el unum argumentum, encuentra su clímax y su punto de retorno - no de quiebre, como en el caso de la entificación divina- y su cierre, en una tematización de la realidad como algo de suyo, y de la inteligencia sentiente como mera actualización de esa realidad. Al Dios de la fe se le puede aplicar -sin pretensiones 
demostrativas sobre su ser y su existencia - la fórmula de que es "[...] algo mayor que lo cual nada puede pensarse [...]" (SAN ANSELMO DE CANTERBURY, 2009, p. 78); pero siempre y cuando se lo piense como realidad fundamental.

\section{LA VÍA dE LA RELIGACIÓN}

La intención es mostrar que entre la tesis del unum argumentum, de San Anselmo, y la del problema de Dios, en el pensamiento de Zubiri, hay una relación hermenéutica circular; es decir, la posibilidad de que una sea interpretada desde la otra, alcanzándose así un ámbito de comprensión mayor que el que tendría cada cual en solitario sobre el asunto. La exposición de las ideas de Zubiri será muy esquemática, y de ninguna manera hace justicia a las densas investigaciones contenidas en El Problema Teologal del Hombre: Dios, Religión, Cristianismo y en El Hombre y Dios.

Para mostrar la relación de continuidad entre San Anselmo y Zubiri sobre el tema de Dios, según cada cual lo aborda, se pueden seguir varios caminos. Uno de ellos es la afirmación de que hay, en la estructura radical de la realidad humana, una dimensión teologal -no teológica-9. ¿En qué consiste esa dimensión teologal del hombre? ¿y por qué con esa dimensión teologal no se ha abandonado el ámbito de la filosofía? Así responde Zubiri: “[...] el logos personal humano es la dimensión por la que el hombre está desde sí mismo y en cuanto hombre vertido al @eós. Esto es lo que temáticamente he llamado dimensión teologal del hombre." (ZUBIRI, 2015, p. 26). Desglosadas, las ideas que deben ser destacadas son las siguientes: a) hay un logos humano personal; b) ese logos conforma una dimensión radical de la realidad humanan; c) en virtud de esa dimensión el hombre está vertido, “[...] desde sí mismo y en cuanto hombre [...]” (ZUBIRI, 2015, p. 26), al @eós; d) la

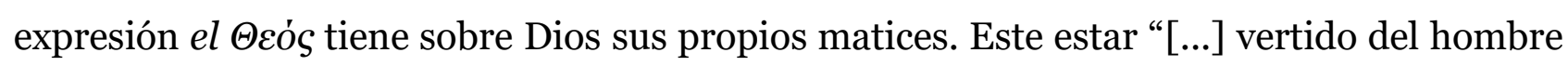
a Dios [...]" (ZUBIRI, 2015, p. 26) es lo que Zubiri entiende bajo los términos de que Dios es un problema para el hombre: el hombre está lanzado $(\pi \rho \circ \beta \dot{\alpha} \lambda \omega \omega)$ hacia Dios, desde su más íntima estructura. ¿Cuál es la diferencia entre lo teologal y lo teológico? Lo segundo pasa por una confesión de fe, y por su institucionalización en una determinada religión, y es el esfuerzo por comprender racionalmente los contenidos de esa fe; lo teologal, en cambio, se refiere a esa respectividad real del hombre en cuanto hombre al $\Theta \varepsilon o ́ s$.

\footnotetext{
9 Esta afirmación descansa, en buena medida, en el resultado de las investigaciones que Zubiri emprendió en Sobre la Esencia (ZUBIRI, 1998, p. 159-161; 242-243).
} 
La tesis fuerte de Zubiri dice, pues, que el hombre está volcado, vertido, desde la estructura más radical de su realidad, a Dios: a "[...] algo mayor que lo cual nada puede pensarse [...]” (SAN ANSELMO DE CANTERBURY, 2009, p. 78), al @eós. Esta versión no depende de su conceptualización; sino, más bien, esta tiene su condición de posibilidad en aquella. No se descubre intelectualmente -o por la fe- a Dios y luego se vierte a él; lo que la intelección descubre es su versión al @eós. De tal suerte es la respectividad y versión humana a lo divino, que el hombre es la manera humana de la realidad de Dios; tal como sucede, también, con toda vida animal y vegetativa. Todas las cosas de este mundo son divinas, cada cual según su forma y modo de realidad. El esfuerzo racional de San Anselmo ha consistido en retrotraerse a la teología -incluso a la confesión de fe-, para hacer contacto intelectual con esa dimensión teologal de la versión y respectividad humana al @eós. La atestación meramente empírica de la presencia del unum argumentum, y de las discusiones que ha suscitado -desde el siglo XI hasta nuestros días-, puede invocarse como señal de ese problematismo de Dios para el hombre, incluso en tiempos que se comprenden a sí mismos como seculares. Pero dado que, según dice Zubiri, "[...] una cosa es la firmeza de un estado de creencia, y otra, su justificación intelectual." (ZUBIRI, 2012, p. 4). Elucidar que el hombre

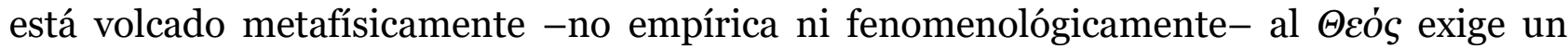
recorrido intelectual que muestre la continuidad, que se ofrece de suyo, entre el logos personal -la dimensión teologal-, momento de la estructura radical del hombre, y la realidad divina.

Se sostiene taxativamente que Dios es un problema $(\pi \rho \circ \beta \dot{\alpha} \lambda \lambda \omega)$ para el hombre. Pero para todo hombre -especialmente para el hombre actual (ZUBIRI, 2015, p. 1-5), piensa Zubiri; y no únicamente para el creyente que debe dar razón de su fe, como hace San Anselmo. Es en la radicalización del problema de Dios de este modo en la que el unum argumentum encuentra un suelo fértil y renovado. ¿Por qué el hombre, respecto del $\Theta \varepsilon o ́ s$, se encuentra en ese carácter problemático? Porque todo hombre -lo haga teóricamente de manera explícita, o no; ilo hace de manera práctica!- se encuentra avocado a estar dando razón de su realidad. Y la respuesta de Heidegger, en el sentido de que el fundamento del ser del ente es que no hay fundamento, se muestra a todas luces insuficiente: como si la cuestión del fundamento pudiera resolverse en esa circularidad que elabora la comprensión entre el Dasein y su ser-en-el-mundo (HEIDEGGER, 2003, p. 109). Porque no es sólo y en primer lugar un problema de comprensión, un problema intelectual; aunque sea la intelección la vía para exhibir ese problematismo último y radical. 
Ese logos personal es un desdoblamiento del logos sentiente, el segundo momento de la estructura de la inteligencia sentiente; y esta es un complejo de notas de la sustantividad humana, de la suficiencia constitucional de su realidad, cuyo acto primario es el mero hacerse presente de lo real en la intelección: el acto radical de la inteligencia no es concebir (Kant), ni abstraer (Aristóteles), ni desvelar (Heidegger), ni tender (Husserl); el acto fundamental es la mera actualidad de lo real en la inteligencia (Cfr. ZUBIRI, 1980, p. 133188), el mero hacerse presente de lo otro en la intelección. Porque lo real se hace presente, se actualiza, en la inteligencia, por eso luego puede concebir, desvelar, tender, etc. En ese primer momento, la inteligencia solo sabe que aquello que se ha hecho presente es real, es decir, es algo de suyo, diferente a la intelección que lo intelige. Es el momento de la mera aprehensión primordial de realidad. Pero la realidad actualizada le exige más a la inteligencia; porque esta quiere saber qué es en realidad aquello que se ha actualizado, que se le ha hecho presente. Saber lo que algo es en realidad no es ya aprehensión primordial, sino logos: la intelección de algo en un campo de realidad, la intelección de una cosa real de una cosa que es de suyo- desde otra cosa real (Cfr. ZUBIRI, 1982). El logos sentiente intelige una cosa real desde otra cosa real. ¿Por qué el logos personal humano es un desdoblamiento del logos? En ese momento de despliegue de la intelección es donde queda inscrito el logos personal humano -el logos que sabe que el hombre es su realidad-por el que la realidad humana está vertida a Dios desde su propia estructura radical: un logos que dice que Dios es “[...] algo mayor que lo cual nada puede pensarse [...]" (SAN ANSELMO DE CANTERBURY, 2009, p. 78); un logos que hila, no dos cosas diferentes, sino que intelige lo absolutamente absoluto -Dios- desde lo relativamente absoluto -el hombre-. La intelección que empieza siendo aprehensión primordial de realidad se despliega en el logos campal, en la intelección de unas cosas desde otras, para saber lo que una cosa es en realidad. En el tercer momento de su despliegue, la intelección quiere saber, no solo que algo es real y lo que en realidad sea eso aprehendido, sino que quiere saber lo que lo real es en profundidad en el mundo: este es el momento de la razón (Cfr. ZUBIRI, 1983).

Los tres momentos del despliegue de la inteligencia sentiente están siempre recubriéndose; porque no se trata de un proceso cronológico, sino de una estructura metafísica. Ninguna realidad puede dar razón de sí misma única y exclusivamente desde sí misma -por ejemplo, desde la mera aprehensión primordial de realidad-, sino que ha de hacerlo desde otras. Y este tener que recurrir a otras realidades para dar razón del sí, que es asunto del logos, es ya un intento por ir a la profundidad de las cosas en su respectividad mundanal. En la afirmación que dice que Dios es “[...] algo mayor que lo cual nada puede 
pensarse [...]" (SAN ANSELMO DE CANTERBURY, 2009, p. 78) ha quedado condensado el despliegue completo de la intelección sentiente: a la inteligencia le es dado, como diferente a ella, la actualización de algo mayor que -... lo cual nada puede ser pensado-, en primer lugar; en segundo lugar, en esa primera aprehensión -pero recubriéndose con ella-, le es exigido un movimiento intelectivo que le lleva desde todo lo que puede ser pensado hasta ese "[...] algo mayor que lo cual nada puede pensarse [...]" (SAN ANSELMO DE CANTERBURY, 2009, p. 78). Pero, en tercer lugar, ese acto primero que aprehende la realidad algo mayor que de manera primordial, lo mismo que el movimiento del logos desde lo que se puede pensar hasta aquello mayor que lo cual nada puede ser pensado, es una marcha de la razón que quiere saber lo que el hombre, en tanto vertido a Dios, es en la profundidad del mundo.

Toda realidad es una estructura sustantiva de notas adventicias, constitucionales y constitutivas, en virtud de lo cual esa realidad es algo de suyo, algo real (Cfr. ZUBIRI, 1998, p. 87-88); y no sólo algo para ser pensado o concebido. Lo que hace a una realidad diferente a otra es el modo como las notas quedan estructuradas. Si bien Heidegger puede tener algo de razón en que el sentido del ser del ente le va al Dasein (HEIDEGGER, 2003, p. 35), ni siquiera él se atreve a decir que la entidad del ente le vaya al Dasein, precisamente porque la realidad del ente es anterior a toda comprensión: las notas de la estructura sustantiva de ese ente que es una mesa, por ejemplo, no dan para envolver un regalo, así lo pretendiera la comprensión de Dasein ${ }^{10}$.

En virtud de esa nota que es la inteligencia sentiente, la realidad humana no solo es algo de suyo, como toda realidad, sino que es suya: el hombre es una realidad que es, cada vez, su realidad. Por este carácter según el cual la realidad humana es su realidad, del hombre se dice que es una persona. Una piedra y un hombre que se precipitan al vacío en un acantilado, no lo hacen de la misma manera; si bien, en ambos casos la caída es real, la diferencia es que, aún en el caso más involuntario, el hombre se tiene que hacer cargo de la realidad de su caída: es una caída de $s u$ realidad. La caída de ese hombre no es sólo una caída de suyo, igual que la de la piedra, sino que es una caída suya, de la propia realidad. Esta suidad -este ser suya- es una de la características exclusivas de la realidad humana.

El hombre es una realidad personal cuya vida consiste en hacer física y realmente (su ser sustantivo) su Yo. La persona humana hace este su ser apoyado en el poder de lo real. Sólo en y por este apoyo puede la persona vivir y ser: es el fenómeno de la religación. La persona

${ }^{10}$ Con la salvedad de que habría que reparar entre cosa real y cosa sentido en la filosofía primera de Zubiri (1998, p. 104-109). 
no está simplemente vinculada a las cosas o dependiente de ellas, sino que está constitutiva y formalmente religada al poder de lo real, el cual constituye eo ipso la fundamentalidad misma de la vida personal. La religación no es mera vinculación ni es un sentimiento de dependencia, sino la versión constitutiva y formal al poder de lo real como fundamento de mi vida personal. (ZUBIRI, 2012, p. 145).

El hombre no hace su realidad; esa simplemente le es dada. Pero tiene que hacer su ser en el mundo, desde las posibilidades de esa realidad; tiene que hacer su Yo. El hombre está irremisiblemente religado a la realidad, depende de ella para la actualización de su ser en el mundo, de su persona. Desde el punto de vista de su realidad, de la suficiencia de las notas que le constituyen, el hombre no puede cambiar nada: tiene una estructura anatómica tal, una estatura determinada, una genética heredada, etc.; pero desde el punto de vista de su estar presente en el mundo, de su realización, está todo por hacer: sobre la conformación de su Yo, de su personalidad, el hombre tiene todo como tarea. Pero para hacerse, para poder realizarse, el hombre está religado irremisiblemente a la realidad, a su poder. El Yo del hombre, su estar presente en el mundo, su persona, está realizándose siempre y sólo desde las posibilidades que le da su religación a la realidad. El poder de lo real, al que le hombre está religado irremediablemente, no lo pone el hombre sino que es anterior a él: la religación del hombre al poder de la realidad no pende en manera alguna de la voluntad humana; sino que es precisamente al revés: la inteligencia, la voluntad y el sentimiento tienen en el poder de lo real tanto sus orígenes y posibilidades como sus límites. "Vivir, poseerse como Yo, es, en todas sus dimensiones, notas y formas, siempre y solo un moverse, un hacerse en el poder de lo real. Por esto es por lo que la religación al poder de lo real es la base de todo nuestro problema y el punto de partida de la discusión.” (ZUBIRI, 1998, p. 147). La fe religiosa de San Anselmo, la que está buscando un anclaje racional, no ha de buscarlo fuera de ella, más allá de ella; la vía es la de la religación (Cfr. SOLARI, 2012, p. 420-428) del hombre a la realidad, condición de posibilidad de toda religión positiva. La experiencia religante al poder de lo real es la base para dibujar "[...] el perfil de la idea misma de Dios. Por esto es por lo que todos estamos de acuerdo con ella no en tanto que realidad, pero sí en tanto que idea.” (ZUBIRI, 2012, p. 148).

La idea de Dios venida de la experiencia del poder de lo real puede condensarse en tres afirmaciones. En primer lugar: “[...] Dios tiene que ser el fundamento del poder de lo real. Por tanto, eo ipso un fundamento último, posibilitante e impelente. Por la vía de la religación, si llegamos a Dios, habremos llegado, pues, a un Dios en tanto que Dios." (ZUBIRI, 2012, p. 148). En segundo lugar: 
[...] Este Dios ha de ser una realidad suprema, pero no un ente supremo [...]. Dios no es un ente divino; es realidad suprema [...]. Dios está allende el ser. Dios no tiene ser; sólo lo tienen las cosas mundanales, las cuales, por ser 'ya' reales, 'son' en el mundo. Como fundamento del poder de lo real, Dios sería formalmente realidad suprema, esto es, última, posibilitante e impelente. (ZUBIRI, 2012, p. 148-149).

Y en tercer lugar:

¿En qué consiste el carácter "supremo" de esta cuestionada realidad? El poder de lo real es el fundamento de la constitución de mi Yo. Y mi Yo, según hemos visto, es algo absoluto. Que mi Yo sea absoluto significa que es Yo "frente" a toda realidad, esto es, frente a la realidad en cuanto tal. Pero este Yo es tan sólo relativamente absoluto porque es un Yo cobrado "frente" a la realidad en cuanto tal; es decir, porque es un absoluto que necesita de esta realidad en cuanto tal para poder ser absoluto. En otro términos: el Yo es absoluto, pero a su modo, relativamente. Si hay un Dios, será una realidad que es el fundamento de mi relativo ser absoluto. Y, por tanto, será realidad absoluta, no a su modo, sino simpliciter. Una realidad que es plenamente real y absoluta no "frente" a la realidad en cuanto tal, sino "en y por sí misma" en cuanto realidad. Es lo que llamaré realidad absolutamente absoluta. "Supremo" significa en este problema "absolutamente absoluto. Y esta sería, de existir, la realidad divina. (ZUBIRI, 2012, p. 149).

Pero Dios no es sólo una idea; es el fundamento del poder de lo real al que todo hombre está esencialmente religado. De esa realidad suprema, absolutamente absoluta, es de la que se puede decir con propiedad, sin sobresaltos, que es "[...] aquello mayor que lo cual nada puede ser pensado.” (SAN ANSELMO DE CANTERBURY, 2009, p. 78).

\section{CONSIDERACIÓN FINAL}

Esta meditación muestra que hay una continuidad hermenéutica, vía la intelección, entre el unum argumentum de San Anselmo y el problema de Dios en Zubiri. Sobre esta circularidad hermenéutica queda la tarea de profundizarla, ampliarla, desarrollarla en sus posibilidades más propias. Las investigaciones de Zubiri aportan un valiosísimo material con la tesis del poder de lo real- para dicha empresa, que tendrá que continuar en otro momento.

\section{REFERENCIAS}

GILSON, É. El ser y los filósofos. Pamplona: EUNSA, 2005.

GOMÉS, M. A. Presentácion. In: SAN ANSELMO DE CANTERBURY. Proslogion: con las réplicas de Gaunilón y Anselmo. Traducción Julián Velarde Lombraña. Madrid: Técnos, 2009. p. 13. 
GONZÁLEZ FAUS, J. I. La humanidad nueva. Ensayo de cristología II. Madrid: Eapsa, 1976.

HEIDEGGER, M. Ser y tiempo. Traducción Jorge Eduardo Rivera. Madrid: Trotta, 2003.

ISAÍAS. In: Biblia de Jerusalén: revisada y aumentada. Bilbao: Desclée de Brouwer, 1998.

KANT, I. Crítica de la razón pura. Traducción Pedro Rivas. Madrid: Santillana, 1997.

KIERKEGAARD, S. La época presente. Traducción Manfred Svensson. Madrid:

Editorial Trotta, 2012.

LÉVINAS, E. Totalidad e infinito: ensayo sobre la exterioridad. Salamanca: Sígueme, 2006.

MARION, J.-L. ¿Es el argumento ontológico realmente ontológico? el argumento sobre la existencia de Dios en San Anselmo y su interpretación metafísica en Kant. Tópicos (México), n. 32, p. 179-205, 2007. Disponible en:

http://www.scielo.org.mx/scielo.php?script=sci_arttext\&pid=So188-

66492007000100179. Acceso en: 14 nov. 2020.

MERLEAU-PONTY, M. Fenomenología de la percepción. Barcelona: Ediciones Península, 2000.

RICOEUR, P. Sí mismo como otro. México: Siglo XXI, 1996.

SALMOS. In: Biblia de Jerusalén: revisada y aumentada. Bilbao: Desclée de Brouwer, 1998.

SAN AGUSTÍN. Obras completas II: las confesiones. Madrid: BAC, 1991.

SAN ANSELMO. Monologion. In: FERNÁNDEZ, C. Los filósofos medievales: selección de textos. Madrid: BAC, 1980, p. 44-111.

SAN ANSELMO DE CANTERBURY. Proslogion: con las réplicas de Gaunilón y Anselmo. Traducion Julián Velarde Lombraña. Madrid: Técnos, 2009.

SANTO TOMÁS DE AQUINO. Suma de teología I. Parte I. Madrid: BAC, 2001.

SOLARI, E. La raíz de lo sagrado: contribuciones de Zubiri a la filosofía de la religión. Chile: RIL editores, 2012.

ZUBIRI, X. Inteligencia sentiente: inteligencia y realidad. Madrid: Alianza - Fundación Xavier Zubiri, 1980.

ZUBIRI, X. Inteligencia y logos. Madrid: Alianza - Sociedad de Estudios y Publicaciones, 1982.

ZUBIRI, X. Inteligencia y razón. Madrid: Alianza - Sociedad de Estudios y 
Publicaciones, 1983.

ZUBIRI, X. Sobre la esencia. Madrid: Alianza Editorial - Fundación Xavier Zubiri, 1998.

ZUBIRI, X. El hombre y Dios: nueva edición. Madrid: Alianza - Fundación Xavier Zubiri, 2012.

ZUBIRI, X. El problema teologal del hombre: Dios, religión, cristianismo. Madrid: Alianza Editorial - Fundación Xavier Zubiri, 2015. 